\title{
MULTI-AGENT BASED SUPPLY CHAIN MANAGEMENT WITH MARKET EMERGENCE PHENOMENON
}

\author{
Toshiya Kaihara \\ University of Marketing and Distribution Sciences, Japan \\ kaihara@umds.ac.jp
}

\begin{abstract}
Supply chain management is categorised into a discrete resource allocation problem amongst several business units, and requires an optimal solution in terms of product distribution. Market-Oriented programming, which integrates market economics concept into multi-agent paradigm, solves product distribution problem by allocating the scheduled resources according to market prices. We formulate supply chain model as virtual market with the aggregation of agents based on the market-oriented programming, and demonstrate the applicability of economic analysis to this framework.
\end{abstract}

\section{INTRODUCTION}

During the last few years the focus has shifted from factory level to enterprise level due to the increasing global presence of the companies. Supply Chain Management (SCM) is now recognised as one of the best means by which enterprises can make instant improvements to their business strategies in virtual enterprise (VE) environment (Fisher, 1994). Sharing of information is central infrastructure to the optimisation of resource allocation (i.e. product distribution) in the supply chain. SCM is generally based on simple TOC (Theory Of Constraints) with throughputbased costing method, and would conduct effective strategies in the enterprise level by DBR(Drum, Buffer and Lope) concept (Goldratt, 1983).

The management of physical product flows amongst the nodes in the supply chain comes under the intensive study of effective operation in SCM. Resource allocation is a quite important operational criterion at workshop level in SCM. As the number of potential business units in the supply chain increases, an effective management on product distribution plays more important role in dynamic environment. Current SCM concept does not deal with the problem, because TOC is nothing to do with the combinatorial optimisation problem in resource allocation.

Recently the utilisation of multi-agent system in large-sized complex system increases (Deneubourg, 1991). Multi-agent paradigm has several characteristics, such as autonomy, pro-activeness, social ability, and emergence. In the paradigm a global goal of the whole system is achieved as the aggregation of their local objectives with their negotiation. In supply chain networks each business unit behaves independently and autonomously with simple goals of achieving myopic views of local optimum. The situation is quite similar to distributed decision making mechanism in multi-agent paradigm, and it is natural to model supply chain 
networks in a multi-agent programming. In such an environment, each agent represents the independent business unit with conflicting and competing individual requirements, and may possess localised information relevant to its utilities. To recognise this independence, we treat the business units as agents, ascribing each of them autonomy to decide how to deploy resources under their control in service of their interests.

Within this model, a distributed SCM can be analysed according to how well it exhibits the following properties:

- Self-interest agents can make effective decisions with local information, without knowing the private information and strategies of other agents.

- The method requires minimal communication overhead.

- Solutions do not waste resources. If there is some way to make some agent better off without harming others, it should be done. A solution that cannot be improved in this way is called Pareto optimal.

Assuming that a resource allocation problem in SCM must be decentralised in considering a practical application, market concept can provide several advantages:

- Markets are naturally distributed and agents make their own decisions about how to bid based on the prices and their own utilities of the goods.

- Communication is limited to the exchange of bids and process between agents and the market mechanism.

- Since agent must back their representations with exchange offers, some mechanism can elicit the information necessary to achieve Pareto and system optima in some well-categorised situations.

Market-oriented programming is a multi-agent based concept to facilitate distributed problem solving. In market-oriented programming we take the metaphor of an economy computing multi-agent behaviour literally, and directly implement the distributed computation as a market price system. In a market-oriented programming approach to distributed problem solving, the resource allocation for a set of computational agents is derived by computing competitive market of an artificial economy (Wellman, 1996), (Kaihara et al., 1999a), (Kaihara, 1999b).

In this paper we formulate supply chain model as a discrete resource allocation problem with supply/demand agents, and demonstrate the applicability of economic analysis to this framework by simulation experiments. Finally we prove the market mechanism can provide several advantages on resource allocation in SCM.

\section{MARKET-ORIENTED PROGRAMMING}

\subsection{Basic Concept}

In economics, the concept of a set of interrelated goods in balance is called general equilibrium. The general equilibrium theory guarantees a Pareto optimal solution at competitive equilibrium in perfect competitive market (Layard et al., 1978). The connection between computation and general equilibrium is not all foreign to economists, who often appeal to the metaphor of market systems computing the activities of the agents involved (Shoven $e t$ al., 1992).

The theory of general equilibrium provides the foundation for a general approach to the construction of distributed planning system based on price mechanism. In this 
approach the constituent planning agents are regarded as suppliers and demanders in an artificial economy. Their individual activities are defined in terms of production and consumption of resources. Interactions amongst agents are cast as exchanges, the terms of which are mediated by the underlying economic mechanism, or protocol.

\subsection{Bidding mechanism}

Market-oriented programming is the general approach of deriving solutions to distributed resource allocation problems by computing the competitive equilibrium of an artificial economy (Wellman, 1996), (Kaihara et al., 1999a). It involves an iterative adjustment of prices based on the reactions of the agent in the market. Bidding mechanism in market-oriented programming is shown in Figure 1.

Let $\operatorname{Pt}(s)$ be the price of resource $s$ at time $t$. ' $f_{t} m s$ ' and ' $g_{t} n s$ ' represent the supply function of supplier $m$ on resource $s$ at time $t$ and the demand function of demander $n$ on resource $s$ at time $t$, respectively.

The bidding mechanism computes an equilibrium price in each separate market. It involves an iterative adjustment of prices based on reactions of agents in the market. Agent $s$ submits supply and demand functions $\left(f_{t} m s\right.$ and $\left.g_{l} n s\right)$ and the auction adjusts individual prices to clear, rather than adjusting the entire price vector by some increment. The mechanism associates an auction with each distinct resource. Agents act in the market by submitting bids to auctions. In this paper bids specify a correspondence between prices and quantities of the resource that the agent offers to demand or supply as a basic study. Given bids from all interested agents, the auction derives a market-clearing price.

Each agent maintains an agenda of bid tasks, specifying in which it must update its bid or compute a new one. The bidding process is highly distributed, in that each agent need communicate directly only with the auctions for the resources of interest. Each of these interaction concerns only a single resource; the auctions never coordinate with each other. Agents need not negotiate directly with other agents, nor even know of each other's existence.

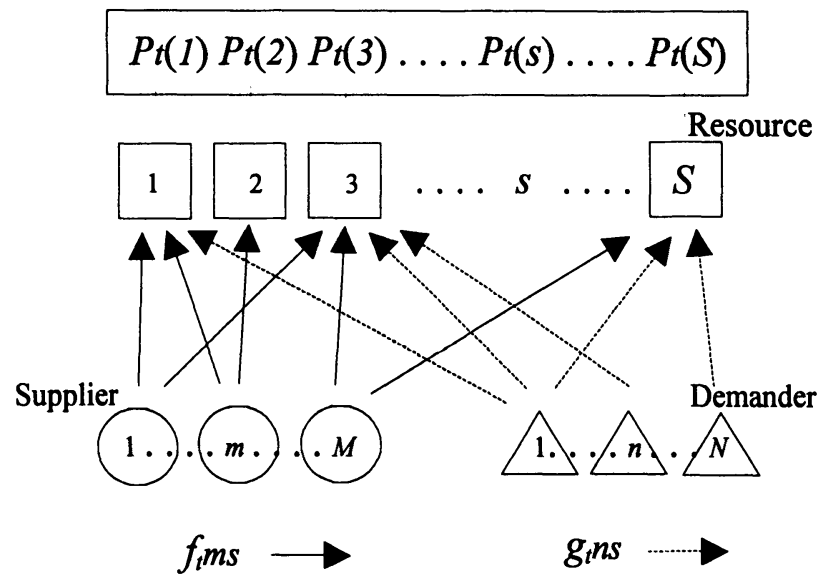

Figure 1 Product Distribution Basic Model 
Agent activities in terms of products required and supplied are defined so as to reduce an agent's decision problem to evaluate the tradeoffs of acquiring different products in market-oriented programming. These tradeoffs are represented in market prices, which define common scale of value across the various products. The problem for designers of computational markets is to specify the strategy by which agent interactions determine prices (Wellman, 1996). Obviously supply chain model is well structured for market-oriented programming, and that means the proposed concept takes advantage of the theory. A Pareto optimal solution, which is conducted by microeconomics, is attainable in resource allocation problem in SCM.

\section{AGENT DEFINITIONS}

\subsection{Preliminaries}

Several variables to formulate agent utilities in this paper are defined as follows:

$x_{k i}$ : Input of resource $i$ in agent $k$

$y_{k j}:$ Yield of resource $j$ in agent $k$

$p_{i}$ : Purchase price of resource $i$ per unit

$P_{i}$ : Sales price of resource $i$ per unit

$E_{k}$ : Profit function of agent $k$

$C_{k}:$ Cost of agent $k$

$S_{k}:$ Sales of agent $k$

${ }_{\max } C_{k}$ : Budget of agent $k$

$l(i, j)(=l)$ :Index of production function from resource $i$ to resource $j$

$f_{k l}: l$ th production function in agent $k$

$x_{k l}$ : Input resource amount into production function $f_{k l}$

$y_{k l}$ : Output resource amount from production function $f_{k l}$

$E_{k l}$ : Profit by production function $f_{k l}$

$c_{k l}$ : Cost by production function $f_{k l}$

$s_{k l}:$ Sales by production function $f_{k l}$

\subsection{Production function}

Suppose supply agent $k$ has a production function $f_{k}$ described in equation (1).

$$
Y_{k}=f_{k}\left(X_{k}\right)
$$

where $X_{k}$ and $Y_{k}$ denote a set of input resources and yield resources in agent $k$ described in equation (2) and (3), respectively.

$$
X_{k}=\left\{x_{k 1}, \Lambda, x_{k m}\right\}, \quad Y_{k}=\left\{y_{k 1}, \Lambda, y_{k n}\right\}
$$

In this paper we adopt Cobb-Douglas function (Layard et al., 1978) as a production function described in equation (4). Since Cobb-Douglas function handles economical scale in the market by index constant $b$, and in $0<b<1$ the production function is defined as a concave down function, in other words, a diminishing returns function. If production function is defined as concave down, market prices are established at a predictable level in the general equilibrium theory. 


$$
y=a x^{b} \quad(\text { where } 0<a, 0<b<1)
$$

Then the production function $f_{k l}$ of agent $k$ for input-output resource set $l(i, j)=l$ is given by

$$
y_{k l}=f_{k l}\left(x_{k l}\right)=a_{k l} x_{k l}^{b_{k l}}
$$

where $x_{k l}$ and $y_{k l}$ denote the amount of input resource $i$ for $f_{k l}$ and the amount of yield resource $j$ for $f_{k l}$, respectively. Then $x_{k i}$ and $y_{k i}$ are defined as

$$
x_{k i}=\sum_{j} x_{k l(i, j)}, \quad y_{k j}=\sum_{i} y_{k l(i, j)}
$$

\subsection{Profit function}

Suppose a set of single unit purchase prices for a resource set $\left\{x_{k 1}, \ldots, x_{k m}\right\}$ is $\left\{p_{1}\right.$, $\left.\ldots, p_{m}\right\}$, and a set of single unit sales prices for a resource set $\left\{y_{k 1}, \ldots, y_{k n}\right\}$ is $\left\{P_{1}\right.$, $\left.\ldots, P_{n}\right\}$, then the total production $\operatorname{cost} C_{k}$ of agent $k$ is defined as

$$
c_{k l(i, j)}=p_{i} x_{k l}, \quad C_{k}=\sum c_{k l}
$$

and the total sales of agent $k$ is defined 'as

$$
s_{k l(i, j)}=P_{j} y_{k l} \quad, \quad S_{k}=\sum_{l} s_{k l}
$$

Then the profit function $E_{k}$ of agent $k$ is finally acquired as

$$
E_{k l}=s_{k l}-c_{k l}, \quad E_{k}=\sum_{l} E_{k l}
$$

\subsection{Budget constraint}

In this paper budget constraint of each agent is considered so as to realise our market model. Suppose the maximum budget of agent $k$ is ${ }_{\max } C_{k}$, then we have

$$
C_{k}=\sum_{l} c_{k l} \leq_{\max } C_{k}
$$

and agent $k$ should behave to maximise its profit $E_{k}$ autonomously.

The basic principle of agents is to maximise their profits under the budget constraints. Their activities should follow the newly proposed theorem, named Profit Maximise Theorem, shown below.

[Theorem] Profit function $E_{k}$ of agent $k$ is maximised by minimised $r_{k}$, which satisfies the following conditions:

$$
\forall l: \frac{\partial E_{k}}{\partial c_{k l}}=r_{k}\left(r_{k} \geq 0\right) \cap \quad C_{k} \leq_{\max } C_{k}
$$

subject to

- $f_{k}$ is differentiable in any $x \in X_{k}$

$$
-\forall l:\left.\frac{\partial f_{k l}}{\partial x_{k l}}\right|_{x_{l}=x}>\left.\frac{\partial f_{k l}}{\partial x_{k l}}\right|_{x_{l}=x+\Delta}
$$

We have the following equation (17) by equation (8), (10), (12), (13).

$$
\begin{aligned}
& \frac{\partial E_{k}}{\partial c_{k l(i, j)}}=\frac{\partial E_{k l}}{\partial c_{k l}}=\frac{\partial}{\partial c_{k l}}\left(s_{k l}-c_{k l}\right) \\
& =\frac{\partial}{\partial c_{k l}}\left[P_{j} f_{k l}\left(c_{k l} / p_{i}\right)-c_{k l}\right] \\
& =\frac{P_{j}}{p_{i}} f_{k l}^{\prime}\left(c_{k l} / p_{i}\right)-1
\end{aligned}
$$


The proof of the theorem is given in Appendix.

\subsection{Supply / demand functions}

Since Cobb-Douglass function shown in (4) is differentiable and

$$
\frac{\partial f_{k l}}{\partial x_{k l}}=a_{k l} b_{k l} x^{b_{k l}-1}>a_{k l} b_{k l}(x+\Delta)^{b_{k l}-1}>0
$$

then the proposed product function (5) perfectly satisfies the conditions (16). Demand function $x_{k l}$, which maximises the agent's profit, is obtained by the Profit Maximise Theorem as follows:

$$
\begin{aligned}
& \frac{\partial E_{k}}{\partial c_{k l(i, j)}}=\frac{\partial E_{k l}}{\partial c_{k l}}=\frac{\partial}{\partial c_{k l}}\left[P_{j} a_{k l}\left(c_{k l} / p_{i}\right)^{b_{k l}}-c_{k l}\right] \\
& \quad=a_{k l} b_{k l} P_{j} c_{k l}{ }^{b_{k l}-1} p_{i}^{-b_{k l}}-1=a_{k l} b_{k l} P_{j} p_{i}^{-1} x_{k l}{ }^{b_{k l}-1}-1=r_{k}
\end{aligned}
$$

Then we have

$$
x_{k l(i, j)}=\left[p_{i}\left(r_{k}+1\right) / a_{k l} b_{k l} P_{j}\right]^{1 / b_{t l}-1}
$$

Supply function $y_{k l}$, which maximises the profit, is also obtained by equation (5), (20) as follows:

$$
y_{k l(i, j)}=a_{k l}\left[p_{i}\left(r_{k}+1\right) / a_{k l} b_{k l} P_{j}\right]^{b_{k l} / b_{k l}-1}
$$

We denote a concrete meaning of the Profit Maximise Theorem. It is obvious to maximise the production function $f_{k l}$ at $r_{k}=0$ by (19), because the function is defined as concave down type. Then agent $k$ has the maximum profit at $r_{k}=0$, if it satisfies the budget constraint ( $C_{k} \leq_{\max } C_{k}$ ). However if agent $k$ breaks the budget constraint $\left(C_{k}>_{\max } C_{k}\right)$, then it has to reduce some amount of input resource $x_{k l}$ to satisfy the constraint. The theorem conducts it should adjust the amount of input resources to have the equivalent value of $\partial E_{k l} / \partial c_{k l}$ in all the production function $f_{k l}$. If the value $r_{k}$ increases, the amount of the demanded resources decreases, and that leads to reduce the cost $C_{k}$. Then the minimised $r_{k}$ in $C_{k} \leq_{\max } C_{k}$ leads to maximise the profit function $E_{k}$.

\section{EXPERIMENTAL RESULTS}

\subsection{Experimental model}

A basic SCM model shown in Figure 2 is prepared to investigate a validity of the proposed approach by computer simulation. The model has a series of three-layered market structure with two-layered agent groups. This model comprises the three types of agent in each layer and three types of good. The interconnectedness of agents and goods defines the market configuration. Comparative analysis of the three market structures reveals the qualitatively distinct economic and computational behaviours realised by the proposed configurations.

Each agent has production functions to transform the resource from market $(M[i][j])$ to market $(M[i+1][j])$, and the parameters are defined as $a[j], b[j]$ in equation (4). The parameters in each agent group are described in Table 1. The parameter $b$ is set in common to each type of the goods, because this parameter is deeply concerned with the price elasticity of the goods. In this figure the outside 
demand function and the outside supply function, which are correspond to sink and source in the experimental model, are defined respectively as

$$
x_{i}=\alpha_{i} p_{i}^{\beta_{i}}\left(\alpha_{i}>0, \beta_{i}<-1\right), y_{j}=\alpha_{j} P_{j}^{\beta_{j}}\left(\alpha_{j}>0, \beta_{j}>0\right)
$$

and each parameter in (22), (23) is described in Table 2.

Table 1. Production function parameters

\begin{tabular}{|l|c|c|c|c|c|c|c|}
\hline & $\mathrm{a}[0]$ & $\mathrm{b}[0]$ & $\mathrm{a}[1]$ & $\mathrm{b}[1]$ & $\mathrm{a}[2]$ & $\mathrm{b}[2]$ & Budget \\
\hline $\mathrm{A}[0][0]$ & 5 & 0.7 & 5 & 0.5 & 5 & 0.3 & 10 \\
\hline $\mathrm{A}[0][1]$ & 3 & 0.7 & 8 & 0.5 & 3 & 0.3 & 10 \\
\hline $\mathrm{A}[0][2]$ & 7 & 0.7 & 7 & 0.5 & 7 & 0.3 & 4 \\
\hline $\mathrm{A}[1][0]$ & 10 & 0.3 & 10 & 0.6 & 10 & 0.4 & 50 \\
\hline $\mathrm{A}[1][1]$ & 4 & 0.3 & 4 & 0.6 & 4 & 0.4 & 100 \\
\hline $\mathrm{A}[1][2]$ & 8 & 0.3 & 4 & 0.6 & 4 & 0.4 & 6 \\
\hline
\end{tabular}

Table 2. Outside production function

\begin{tabular}{|l|c|c|c|c|c|c|}
\hline & $\alpha[0]$ & $\beta[0]$ & $\alpha[1]$ & $\beta[1]$ & $\alpha[2]$ & $\beta[2]$ \\
\hline Supply function & 100 & 1.5 & 100 & 1.5 & 100 & 1.5 \\
\hline Demand function & 100 & -2 & 100 & -2 & 100 & -2 \\
\hline
\end{tabular}

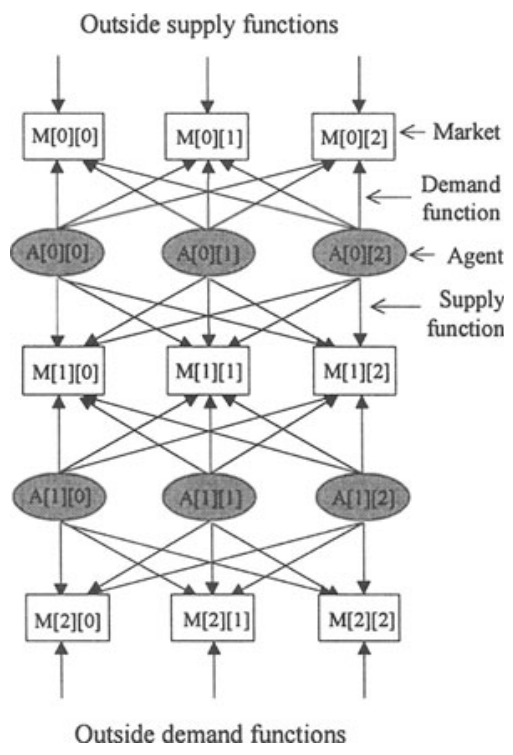

\subsection{Market dynamism}

Figure 2 Supply chain model

Dynamical changes of i) the amount of dealing goods in supply and demand, ii) the prices of the goods, at each layer in the market structure are shown in figure 3, figure 4, respectively.

First of all, it is obvious that both the amount of dealings and the prices are converged into equilibrium in these figures. Since our methodology is perfectly endorsed by 'general equilibrium theory' in the competitive market, we can get a Pareto optimal solution in the equilibrium. That means the goods distribution policy followed by the acquired solutions, i.e. the amounts and the prices, are Pareto optimal in the entire market. Efficient SCM with market mechanism are attainable by the proposed approach.

Secondly it is observed that the number of iterations required to reach equilibrium seems to rise with the price elasticity. For example, Market [0][0] with 0.7 in price elasticity takes longer time to converge than Market [0][2] with 0.3 in price elasticity in figure 3(a). We attribute this to the natural characteristic of price elasticity, and the experimental values completely agree with the general theory in market economics.

Finally it takes longer time to converge into the equilibrium at the market in the middle layer, Market [1][..], compared with the other markets, Market [0][..] and Market [2][..]. This observation is explained by the following reason. We applied the functions described in (22) and (23) as outside functions in Market [2][..] and 
Market [0][..], and they are defined as static functions in the experimental model. On the other hand, Market [1][..] is operated by supply and demand agents with dynamic utility functions. As a result, Market [1][..] behaves dynamically and is more sensitive to trading situation in the competitive market.

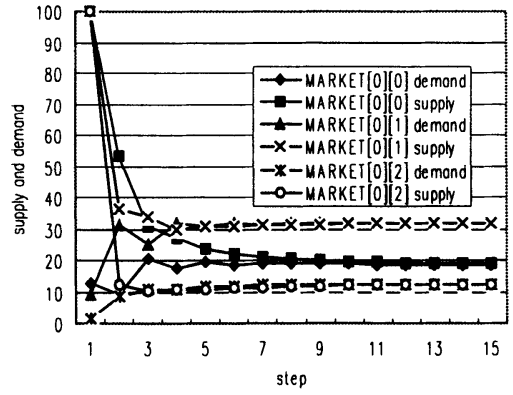

(a) Market[0][ $\square$ F

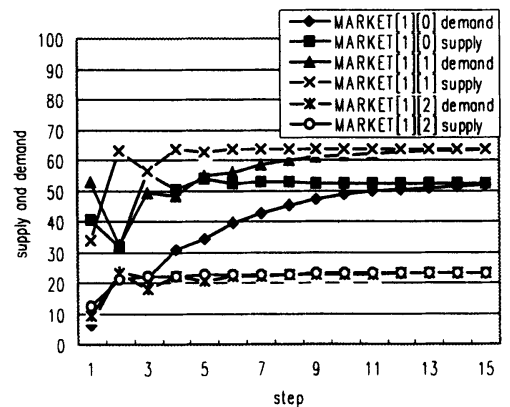

(b) Market[1][ᄆ F

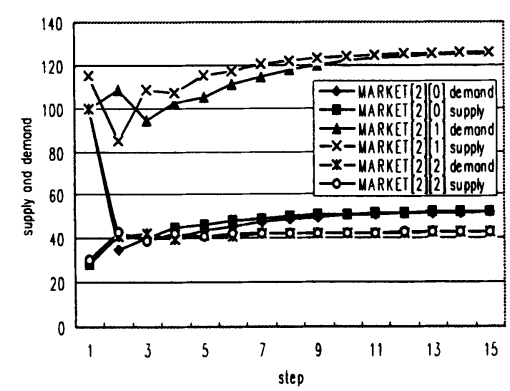

(c) Market[2][0 F

Figure 3 Supply and demand oscillation

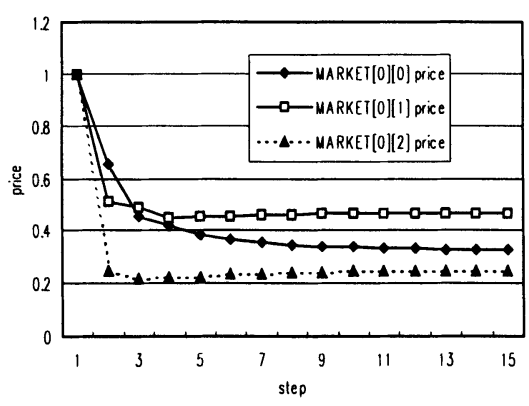

(a) Market[0][0 F

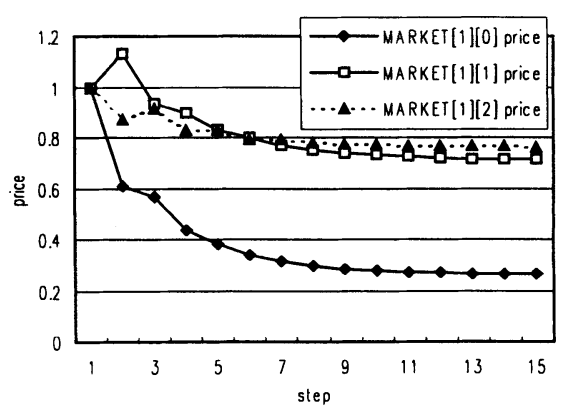

(b) Market[1][ ₹

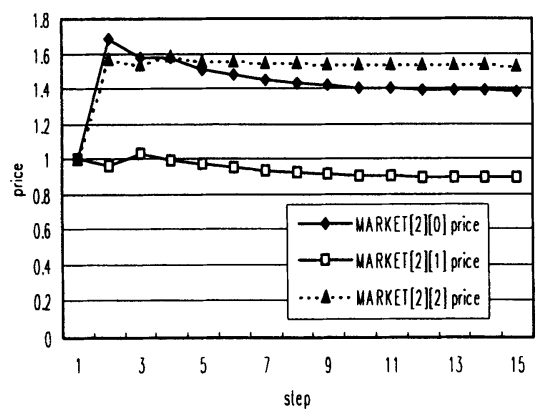

(c) Market[2][ $\mathbf{F}$

Figure 4 Price oscillation

\subsection{Market equilibrium}

The market equilibrium dynamism should be explained by our market definition. The comparison between figure 3(a) and figure 4(a) shows us that the good with larger trading amount has higher price in the equilibrium in Market [0][..]. That is because we defined the function shown in (23) as outside supply function, which characterises positive correlation between the dealing amount and the price. On the 
other hand, the good with larger trading amount has lower price in the equilibrium in Market[2][..] shown in figure 3(c) and figure 4(c). The outside demand function defined in (22), which has negative correlation between the dealing amount and the price, influences the equilibrium. These experimental values are perfectly explicable by our market formulation. The middle layered market, Market [1][..] has more complex dynamism in figure 3(b) and figure 4(b). The dynamism is emerged and explained by the agent utility parameters shown in Table 1 . In this case, Market [1][1] is high both on the dealing amount and on the price in the equilibrium. A set of Agent [0][..] has to offer higher sales price to increase their profit, because $b[1]$ in Agent [1][..] $(=0.6)$ is the highest amongst $b[.$.$] in the second layer, but b[1]$ in Agent $[0][.].(=0.5)$ is lower than $b[0]$ in the first layer.

It has been clarified that all the experimental values in the complex SCM model are perfectly explicable by our market formulation. The experimental results agree with the theoretical trends of perfect competitive market in microeconomics. It is obvious that each market is perfectly competitive and holds market mechanism in general equilibrium.

\section{CONCLUSIONS}

In this paper we proposed a SCM with market economics. We formulated SCM as distributed resource allocation systems, based on general equilibrium theory and competitive mechanism. The approach works by deriving the competitive equilibrium corresponding to a particular configuration of agents and markets. After defined production functions, we introduced budget constraint for practical use and newly proposed Profit Maximise Theorem as an agent strategy. It has been confirmed by simulation experiments that careful constructions of the decision process according to economic principles can lead to efficient distributed resource allocation in SCM, and the behaviour of the system can be analysed in economic terms.

The contribution of the paper lies in the idea of SCM based on market-oriented programming, an algorithm for distributed computation of competitive equilibria of computational economics, and an initial illustration of the approach on a simple SCM. Effective SCM in global environment is expected by this research.

\section{Acknowledgements}

This research is supported by International Research program IMS(Intelligent Manufacturing System) of MITI Japan, under contract No.9919 (HUTOP project).

\section{REFERENCES}

Deneubourg, J. (1991). The dynamics of collective sorting robot-like ants and ant-like robots, Proc. of the First Int. Conference on Simulation of Adaptive Behavior, The MIT Press.

Fisher M. L. (1994). Making supply meet demand in uncertain world, Harvard Business Review, May/Jun. Goldratt E. M. (1983). The GOAL, North River Press.

Kaihara T. and Namikawa J. (1999a). A Study on Distributed Resource Allocation by Using MarketOriented Programming in Distribution System, The Transactions on The Institute of System, Control and Information Engineers, Vo.12, No.6, pp.349-356.

Kaihara T. (1999b), Supply Chain Management with Market Economics, Manufacturing for a global market, M. T. Hillery \& H. J. Lewis Eds, Vol. 1, pp.659-662.

Layard, P. R. G. and Walters, A. A. (1978). Microeconomics Theory, London, McGraw-Hill. 
Shoven J. B. and Whalley J. (1992). Applying General Equilibrium, Cambridge University Press.

Wellman M. P. (1996). A Market-Oriented Programming Environment and its Application to Distributed Multi-commodity Flow Problems, Proc. of ICMAS-96, pp385-392.

\section{APPENDIX}

[Theorem] Profit function $E_{k}$ of agent $k$ is maximised by minimised $r_{k}$, which satisfies the following conditions:

[Proof]

$$
\forall l: \frac{\partial E_{k}}{\partial c_{k l}}=r_{k}\left(r_{k} \geq 0\right) \quad \bigcap \quad C_{k} \leq_{\max } C_{k}
$$

Note that

$$
\frac{\partial f_{k l}}{\partial x_{k l}}=\frac{\partial f_{k l}}{\partial c_{k l}} \cdot \frac{\partial c_{k l}}{\partial x_{k l}}=p_{i} \frac{\partial f_{k l}}{\partial c_{k l}}
$$

then

Also, note that

$$
\left.\frac{\partial f_{k l}}{\partial c_{k l}}\right|_{c_{l l}=c}>\left.\frac{\partial f_{k l}}{\partial c_{k l}}\right|_{c_{H^{\prime}=c+\Delta}} \quad \text { in any positive value } \Delta
$$

$$
E_{k l}=P_{j} f_{k l}\left(x_{k l}\right)-p_{i} x_{k l}=P_{j} f_{k l}\left(c_{k l} / p_{i}\right)-c_{k l}
$$

then $E_{k l}$ is regarded as concave down by (A3). $E_{k l}$ is maximised with the condition (A5).

$$
\forall l: \frac{\partial E_{k}}{\partial c_{k l}}=0
$$

Let $C^{\prime}{ }_{k}$ denote total expense in (A4) and if $C^{\prime}{ }_{k} \leq_{\max } C_{k}$, then the maximum profit is given with (A5). Otherwise the maximum profit is not given with (A5) due to the budget constraint. If $C^{\prime}{ }_{k}>{ }_{\max } C_{k}$, agent $k$ must consider to increase $C_{k l}$ by $\Delta c$, and reduce $C_{k l}$, by $\Delta c$ shown in (A6), (A7).

$$
\begin{aligned}
& \left.\frac{\partial E_{k}}{\partial c_{k l}}\right|_{c_{k l}=c+\Delta c}=\frac{P_{j}}{p_{i}} f_{k l}^{\prime}\left[\left(c_{k l}+\Delta c\right)\right]-1=r_{k l} \\
& \left.\frac{\partial E_{k}}{\partial c_{k l^{\prime}}}\right|_{c_{k^{\prime}}=c-\Delta c}=\frac{P_{h}}{p_{g}} f_{k l^{\prime}}^{\prime}\left[\left(c_{k l^{\prime}}-\Delta c\right)\right]-1=r_{k l^{\prime}}
\end{aligned}
$$

then we obtain that $r_{k l}<r_{k}<r_{k l^{\prime}}$ from (A3).

Let $\Delta E_{k l}$ and $\Delta E_{k l}$ denote the increased profit in $f_{k l}$ and the diminished profit in $f_{k l}$, respectively, then

$$
\Delta E_{k l}=\alpha \Delta c \quad\left(r_{k l}<\alpha<r_{k}\right), \quad \Delta E_{k l^{\prime}}=\beta \Delta c\left(r_{k}<\beta<r_{k l^{\prime}}\right)
$$

It is obtained that $\Delta E_{k l}<\Delta E_{k l}$ from (A8), and that means the diminished profit is greater than the increased profit in any $\Delta c$.

Therefore the profit function $E_{k}$ of agent $k$ is maximised with the condition

$$
\forall l: \frac{\partial E_{k}}{\partial c_{k l}}=r_{k} \quad\left(r_{k} \geq 0\right) \quad \text { in } \quad C_{k} \leq_{\max } C_{k}
$$

Finally, $r_{k}$ is minimised with the condition (A9), since the profit function $E_{k}$ is concave down. This completes the proof. 